\title{
Ring nebulae: what they tell us about Wolf-Rayet stars
}

\author{
Anthony P. Marston \\ Physics \& Astronomy Department, Drake University, \\ Des Moines, IA 50311, USA
}

\begin{abstract}
The environments of evolved massive stars provide an opportunity of obtaining information on the past, as well as current, condition of the stars themselves. In this review we will look at the incidence of ring nebulae around Wolf-Rayet stars, their differing morphologies at various wavelengths and the existence of multiple, concentric shells. We use this information to show that WRs are indeed evolved stars and that the various phases of evolution for a WR star are evidenced in their environments. Abundance measurements and kinematics show that complex forms of mass ejection are likely to have occurred in the evolution of WR stars providing clumpy structures of dust, and both ionized and neutral gas. Gas kinematics also provide estimates to the timescales of each of the evolutionary phases of WR stars, which can be combined with estimates of nebular masses to provide the approximate values for such crucial parameters as total mass-loss and historical mass-loss rates. Overall, it is illustrated that studies of the environments of WR stars have the potential to provide important information about the mass-loss history of very massive stars, including estimates of the time period of each mass-loss phase, typical mass loss rates, total mass lost and likely evolutionary path. Some of the remaining problems relating to the use of ring nebulae as probes to the evolutionary history of WR stars are also discussed.
\end{abstract}

\section{Introduction}

The gaseous environments of stars provide the opportunity for studying stellar history, the current state of circumstellar environments being predominantly a function of the output from the central stars. By studying the environments of Wolf-Rayet stars we may therefore reasonably expect to be able to provide insight into the evolutionary history of such stars. We may also provide constraints on evolutionary models.

Current models for single star evolution suggest that the progenitors for WR stars are massive $\mathrm{O}$ stars, which, following a period of approximately $1-3 \times 10^{6}$ years on the main sequence, evolve through at least one phase of major massloss such as a Red Supergiant and/or a Luminous Blue Variable phase before becoming WR stars (e.g., Humphreys 1991; Maeder \& Meynet 1994; Stothers \& Chin 1996). During the O star phase, mass is lost in a fast wind $\left(\sim 2000 \mathrm{~km} \mathrm{~s}^{-1}\right)$ while larger amounts of mass are lost at slower speeds in the intermediate ejecta phase. Alternatively, one or more eruptions may occur in an intermediate LBV phase. The effects of the changing stellar output are expected to be visible in the circumstellar material. 


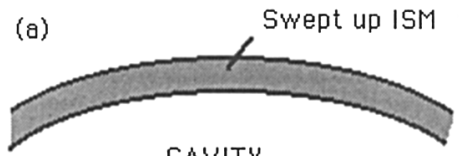

CAVITY

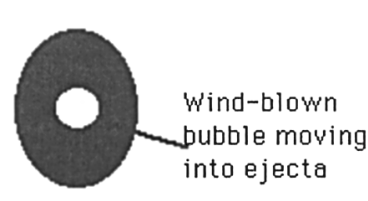

(b) Swept up ISM

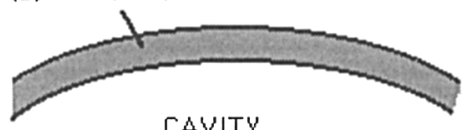

CAVITY

Wind-blawn bubble

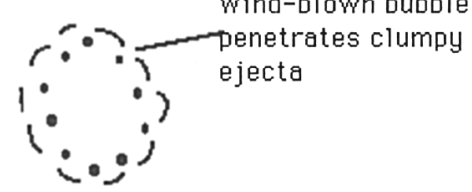

Figure 1. Schematics of evolving ring nebulae. (a) In the early stages of the WR wind phase, a wind-blown bubble is contained within the ejecta which resides in a cavity created by the progenitor $\mathrm{O}$ star. (b) As the wind progresses it moves out beyond the ejecta nebula, penetrating through the gaps between ejecta clumps.

If we consider, for example, a star that evolves from O to RSG to WR star in a reasonably dense gaseous environment, we would expect to see a large cavity produced by the stellar wind of the $\mathrm{O}$ star phase, interior to which would reside material ejected in the RSG phase (e.g., D'Ercole 1992). If the mass-loss during the RSG phase was reasonably constant, then we would expect a $r^{-2}$ density distribution in stellar ejecta around the star. The fast wind from the WR star that follows the RSG phase would then form a relatively fast, expanding windblown bubble inside the ejecta. The three phases would then be visible as three separate shells (Marston 1995; see Fig. 1). At later stages the wind-blown bubble may penetrate out of the RSG ejecta into the cavity produced by the $\mathrm{O}$ star.

A similar scenario may be considered for the case of a LBV as the intermediate stage of evolution. LBVs lose mass through continuous mass-loss and eruptions (Leitherer 1997). If LBVs are an intermediate stage for becoming WR stars, then the circumstellar regions of WR stars may be expected to show evidence of prior eruptions as well as a continuous mass outflow.

In this presentation, we consider the detailed studies that have been made of the circumstellar regions of WR stars and what these observations can tell us about the past history of these stars. We show that the environments predicted from stellar models are consistent with observations, although some adaptations may be required to explain some of the most recent observations.

\section{Optical and infrared morphologies}

Large-scale features around WR stars associated with their O star phase have been noted in many of the $\mathrm{HI}$ observations made by the Argentinian group (e.g., Arnal 1992; Cappa de Nicolau et al. 1988; Dubner et al. 1990). These observations have shown the existence of $\mathrm{H}$ I cavities, that can be several degrees across, with expansion velocities of $6-20 \mathrm{~km} \mathrm{~s}^{-1}$ (see also Arnal et al. these Proceedings). An IRAS survey of all galactic WR stars by Marston (1996) showed that approximately $45 \%$ of all galactic WR stars showed distinguishable shells of 
$>20$ '(also see Manchado et al. 1995). If the majority of these can be attributed to the effects of a progenitor O star phase, then they imply that WR stars have an O star phase of $0.5-10$ million years (Marston 1996). Alternatively, Nichols \& Fesen (1994) suggested that some of these large shells may be associated with high-velocity ejections from a Roche lobe overflow in a binary system or from a supernova blast. However, the slow $\mathrm{H}$ I expansion velocities observed indicate it is more likely that these large shells are indeed the relics of bubbles produced in the O star progenitor-phase of WR stars.

The optical morphologies and kinematics of ring nebulae were characterized by Chu and coauthors in a series of papers (e.g., Chu 1981; Chu \& Treffers 1981; Chu 1982; Chu et al. 1983), who identified three ring nebula types: $R$ type nebulae have large time-scales for formation; E-types are more clumpy nebulae; while $W$-types are formed by the wind of the WR star (see review by Chu 1991). In recent years, the use of wide-field narrow-band CCD imaging has provided improved knowledge of the immediate circumstellar regions of WR stars in the galaxy and Magellanic Clouds (Miller \& Chu 1993; Marston et al. 1994a; Marston et al. 1994b; Dopita et al. 1994; Marston 1997). Studies of the immediate circumstellar environments are expected to provide information on the more recent events in the evolution of WR stars. In particular, the RSG/LBV ejecta and WR wind phases. In total, nearly $50 \%$ of galactic WR stars have been shown to have evidence of an optical ring nebula. Those around WC stars appear slightly larger than WN stars, which suggests the nebulae have, in general, expanded for longer periods of time (Marston 1997). This is consistent with an evolution from WN to WC subtypes expected from single star theories (e.g., Maeder \& Meynet 1994).

The optical appearance of ring nebulae differs in various emission-lines. Observations by Miller \& Chu (1993) show the strong ring nebula NGC 6888, around the star WR 136, has an [OIII] morphology distinct from that observed
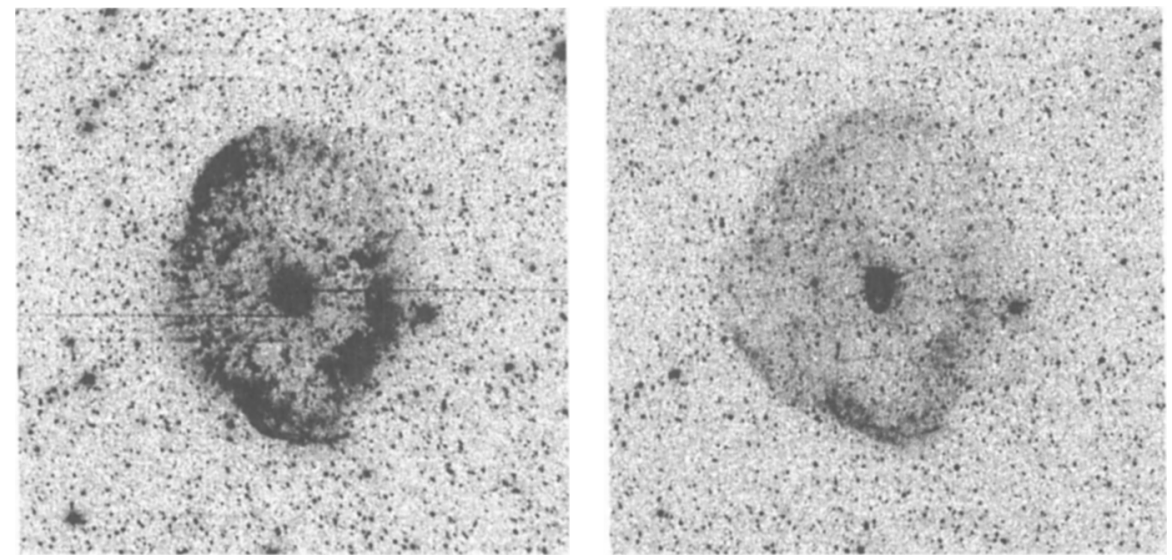

Figure 2. $\mathrm{H} \alpha$ (left) and [OIII] (right) images of the ring nebula RCW 58 around WR 40. The [OIII] image suggests the wind blown bubble from the WR wind has moved out into the cavity beyond the clumpy ejecta, as in Fig. 1(b). 

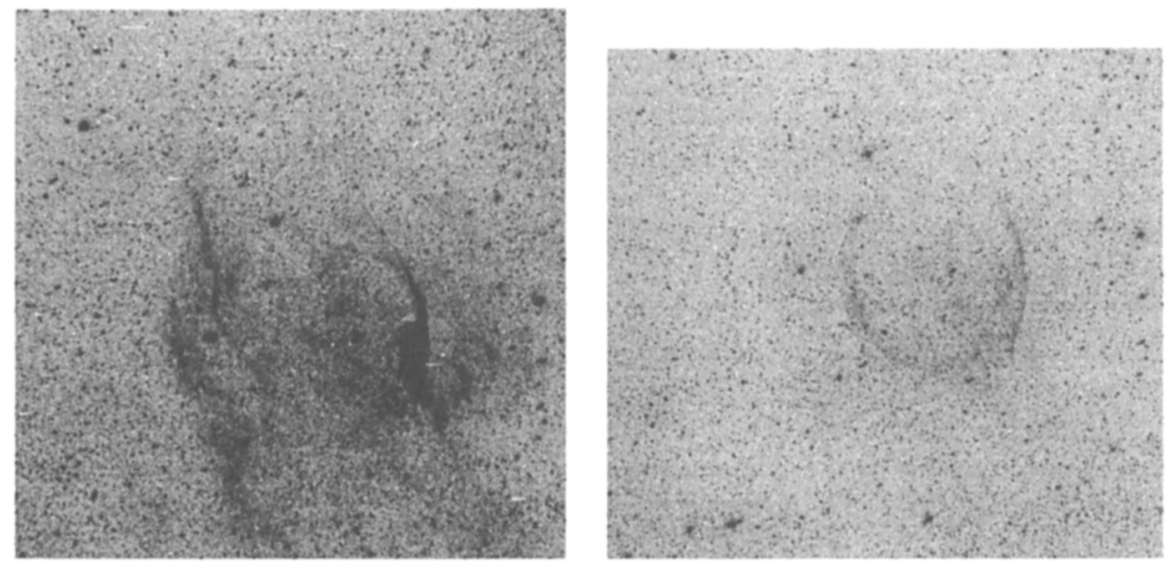

Figure 3. $\quad \mathrm{H} \alpha$ (left) and [OIII] (right) images of the ring nebula RCW 104 around WR 75. The [OIII] image suggests the wind blown bubble from the WR wind is still sweeping up material in the inner portion of the ejecta nebula, as in Fig. 1(a).

at $\mathrm{H} \alpha$. Filaments of [OIII] emission appear external to the bright $\mathrm{H} \alpha$ rim of the nebula. A similar phenomenon is observed in the nebula RCW 58 around WR 40 (see Fig. 2). For the nebula RCW 104 around WR 75, however, the [OIII] makes a spherical shell interior to the strong $\mathrm{H} \alpha$ filaments (see Fig. 3). Dufour $\&$ Buckalew (these Proceedings) suggest that regions of high [OIII] $/ \mathrm{H} \alpha$ ratio in NGC 6888 are produced by partially-radiative strong shocks rather than via photo-ionization (which dominates the rest of the nebula). Such a shock is likely associated with the fast wind-blown bubble, produced in the WR phase of the star, punching through surrounding ejecta from an earlier RSG or LBV phase. A similar situation must exist in RCW 58 (Marston 1995). A natural explanation for the interior shell noted in RCW 104 is therefore that the WR wind-blown bubble, identified by the [OIII] shell, has not had time to progress through the whole ejecta nebula.

A further connection between strong $[\mathrm{OIII}] / \mathrm{H} \alpha$ and the fast WR wind is noted for nebulae around WR stars in M 33 (Marston, Drissen \& Godbout, these Proceedings), where fast expansion is only noted in regions where this ratio is high. This also indicates that a large fraction of [OIII] emission is likely to be collision-induced rather than the result of photo-ionizing flux (also see Dufour \& Buckalew, these Proceedings).

In many cases more than one ring is observed around WR stars in optical and infrared emission. Large $(>20 \mathrm{pc}$ across) rings associated with the progenitor O star phase, an ejecta ring associated with the intermediate RSG or LBV phase, and a wind-blown bubble associated with the WR wind (Marston 1995). This can sometimes coincide with the ejecta shell when the wind-blown bubble penetrates the outer edge of the ejecta, as appears to be the case in the nebulae NGC 6888 and RCW 58. The large rings, ejecta rings and WR wind-blown rings correspond approximately to the $R-, E$ - and $W$-type ring nebula morphologies of Chu (1991). 


\section{Ring nebula abundances}

In a series of papers, Esteban et al. (1991-3) have studied the abundances of WR ring nebulae. In almost all cases nebulae show an enhancement in nitrogen content and helium fraction. Some deficiency is noted in O, although this may be a miscalculation due to the presence of shock excitation (see Dufour \& Buckalew, these Proceedings). Comparisons to stellar surface abundances from evolutionary models (e.g., Maeder, 1990), suggest that the observed abundances in the nebulae are consistent with having been formed from the surfaces of $25-40 \mathrm{M}_{\odot}$ stars at the end of their RSG phase.

Similarities exist between the abundances observed in WR ring nebulae and the ring nebulae around LBV stars (Smith 1995). The similarities between the two types of ring nebulae suggest a common origin.

\section{Neutral gas components}

In Van Buren (1986) the suggestion was made, on the grounds of ring nebula dynamics, that there was a distinct possibility of neutral material in several WR ejecta ring nebulae. In studies of the WR ring nebula NGC 6888, Marston \& Meaburn (1988) illustrated that an estimate of gas mass from IRAS data modeling and a mass estimate based on ring kinematics were in good agreement $\left(\sim 29 \mathrm{M}_{\odot}\right)$, and suggested that the ionized gas mass in the nebula was only a fraction of the total.

Relatively little is currently known about neutral material in WR ring nebulae. Schneps et al. (1981), Gruendl \& Chu (1998) and St-Louis et al. (1998) have shown the presence of molecular gas in the ring nebula NGC 2359 around the star WR 7. Studies of IRAS-HIRES maps by Marston (1997) show cool dust

Figure 4. The ring nebula RCW 58 seen in IRAS-HIRES images showing its clumpy structure. Emission is coincident with $\mathrm{H} \alpha$ emission, but not [OIII] (see Figs. 2a and b). 


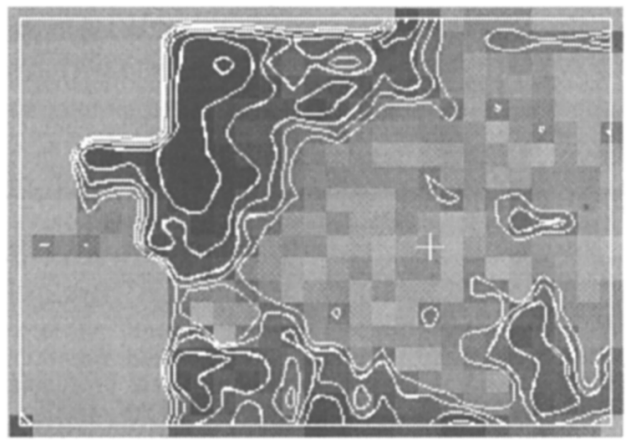

Figure 5. $\quad{ }^{12} \mathrm{CO} 1 \rightarrow 0$ intensity map of the ring nebula around WR 16 showing an almost complete ring around the central star (marked by a cross) with an inner radius of approximately $3 \mathrm{pc}$. The field is approximately $16^{\prime} \times 8^{\prime}$.

clumps are formed in ejecta, in regions closely associated with strong $\mathrm{H} \alpha$ emission (see Fig. 4). It is likely that this clumping of ejecta material is what allows the fast wind from the WR star to penetrate several ring nebulae to form extended [OIII] filaments (e.g., RCW 58: Marston 1995; NGC 6888: Miller \& Chu 1993). An $\mathrm{H}$ I shell has been noted in low resolution observations of the ring nebula S 308 around WR 6 by Arnal \& Cappa (1996) and there is significant neutral hydrogen around and associated with NGC 6888 (Cappa et al. 1996).

In recent ${ }^{12} \mathrm{CO} 1 \rightarrow 0$ emission-line observations of two southern hemisphere ring nebulae significant amount of molecular gas have been found associated with the nebula around the WR star WR 16 (see Fig. 5) and in RCW 104 around WR 75 (also see Marston et al. these Proceedings). Estimated molecular gas mass in the nebula around WR 16 ranges from 4 to $78 \mathrm{M}_{\odot}$ depending on chemical enrichment and uncertain distance (Marston et al. 1999a). Optical spectra suggest $N$ enhancements in both the inner nebula around WR 16 of $2-3$ pc radius, and in outer filaments that appear to bound the region of molecular gas, suggesting the molecular gas is formed in material ejected from the star.

Molecular gas in the nebula RCW 104 around WR 75 shows a very clumpy morphology, with distinct variations in velocity. A system of clumps exist at the systemic velocity, while other molecular gas clumps appear to be moving at $\sim 50 \mathrm{~km} \mathrm{~s}^{-1}$ relative to systemic. This puts them in the same velocity range as high-velocity, nitrogen-rich clumps previously observed by Goudis et al. (1988) in the same part of the nebula. Therefore the fast-moving clumps are CNO rich.

\section{Evolutionary implications}

The surroundings of WR stars are expected to be a function of their evolution, with the effects of different phases appearing in an 'onion shell' form around the stars.

\subsection{Total age and $O$ star phase}

The large-scale features around galactic WR stars seen in IRAS and H I data suggest that, with expansion velocities of typically $10 \mathrm{~km} \mathrm{~s}^{-1}$, time-scales for 
formation of a few million years (Marston 1996). With the relatively uncertain distance to individual WR stars, we are unable to be more precise. The estimated range of formation times of the outer shells are expected to be indicative of the total age of the central WR stars. Most of the period up to the WR stage is expected to be as an $\mathrm{O}$ star. Results are consistent with the $\mathrm{O}$ star age ranges for WR progenitors predicted by Maeder \& Meynet (1994).

\subsection{Intermediate stages of evolution}

Some speculation exists as to the exact nature of the intermediate mass-loss phases of WR stars. Authors have considered various intermediate evolutionary paths, usually including RSGs and/or LBVs. The abundance studies strongly suggest the existence of red supergiant materials in the ejecta nebulae. The neutral gas and IRAS measurements, noted above, also suggest these ejecta have significant amounts of gas (tens of solar masses).

In recent observations, Marston et al. (1999b) have shown the nebula around the LBV star AG Car to have an associated slow outflow of molecular gas $\left(\sim 10-15 \mathrm{~km} \mathrm{~s}^{-1}\right)$. The optical ring nebula around AG Car is expanding at a much faster speed. It is suggested that the flow is the relic of a mass-loss phase prior to the star's current LBV phase. Also, fast-moving CO clumps have been noted in RCW 104 around WR 75, which perhaps would suggest a superposition of expelled molecular clumps on a smoother ejecta. A similar situation may exist in M1-67 around WR 124 (see Grosdidier et al. these Proceedings).

The suggestion from the ejecta material around WR and LBV stars is that a, possibly brief, RSG heavy mass-loss rate phase preceded a LBV in several cases, the two phases providing both smooth and clumpy ejecta structures. Such an evolution has been suggested by Langer (1991) and Stothers \& Chin (1996).

\subsection{Mass-loss in the intermediate phase of evolution}

From the previous section we may conclude that the mass of gas immediately surrounding (typically $2-5$ pc in radius) galactic WR stars is predominantly composed of ejecta material. Mass estimates using IRAS measurements generally suggest nebulae masses of tens of solar masses (Marston 1991; Mathis et al. 1992; Marston 1997). More recently, the existence of molecular and atomic gas components in ring nebulae also indicate that significant gas mass may be found in the neutral gas component of ring nebulae (Arnal \& Cappa 1996; Marston et al. these Proceedings). Mass estimates are restricted by our knowledge of the distance to many galactic WR stars as well as parameters such as assumed dust model (for IRAS measurements), excitation temperature (for CO measurements) and volume filling factor (for ionized gas mass estimates).

\subsection{Wind-blown bubble kinematics and ring mass}

An alternate method might be to consider the kinematics of a wind-blown bubble produced by the fast wind of the WR stage that follows the ejecta phase and forms an expanding bubble inside the ejecta. Following the theory of Dyson (1989), it can be shown that, if we consider ejecta to have a $r^{-2}$ distribution around WR stars, as would be the case for a constant mass-loss through the 
ejecta phase, then the ejecta mass, $M_{e j}$, can be given by

$$
M_{e j}=\frac{16 L_{w}}{81 v_{W B}^{3}} R_{e j}
$$

where $v_{W B}$ is the expansion velocity of the wind-blown bubble, $L_{w}$ is the luminosity of the WR wind and $R_{e j}$ is the radius of the ejecta shell (Marston 1995; see also Arthur, Henney \& Dyson 1996). Using typical parameters for WR stars Marston (1995) illustrated that this implies several tens of solar masses likely exist in nebulae for which expansion velocities are known. This is consistent with results from IRAS modeling (Marston 1991). Note that, with a $r^{-2}$ mass distribution, the expansion speed of the WR wind-blown bubble should be constant, which indicates that the length of time for the WR period is simply given by the radius over velocity of the wind-blown bubble.

An upper limit to the period of major WR mass-loss may be placed by considering the radius of the ejecta nebula and an expansion rate of $15 \mathrm{~km} \mathrm{~s}^{-1}$ from a RSG wind. This suggests ring nebulae were formed in $2 \times 10^{5}$ years, but quite possibly in less if faster expansion occurred in a LBV phase. In order to produce $40 \mathrm{M}_{\odot}$ of ejecta would therefore require a mass-loss rate of $2 \times 10^{-4} \mathrm{M}_{\odot} \mathrm{yr}^{-1}$.

Hydrodynamical simulations by García-Segura et al. (1996a, 1996b) have been performed for evolution involving WR winds penetrating both RSG and LBV ejecta shells. General morphologies of ring nebulae are well reproduced (see García-Segura, these Proceedings).

\section{Conclusions}

Studies of the complex shell systems around WR stars provide clues to the past history of the stars. Current results suggest that WR stars are indeed evolved from massive $\mathrm{O}$ stars that lasted a few million years.

Both abundance measurements and kinematic results suggest that a progression through both a RSG and a LBV phase occur. This causes some problems observationally in that no high mass $\left(>40 \mathrm{M}_{\odot}\right)$ RSG stars have ever been observed (Humphreys-Davidson limit, Humphreys \& Davidson, 1979). Possibly, such objects are highly obscured or similar to the transitional objects IRC+10 420 and HD 17891 (Oudmaijer et al. 1996; Kastner \& Weintraub 1995). This suggests that WR evolution requires the sequence $\mathrm{O} \rightarrow \mathrm{RSG} \rightarrow \mathrm{LBV} \rightarrow \mathrm{WR}$ at least. Ring nebulae also suggest the subtype evolution of WN to WC.

The total mass lost in the more prominent ring nebulae (e.g., NGC 6888 and RCW 58) appears to be several tens of solar masses and significant mass in several ring nebulae is in neutral atomic or molecular gas form.

Major problems remain to be solved. The total mass in ring nebulae is not well known. Very few $\mathrm{HI}$ and CO measurements have so far been made and expansion velocities are known only for a handful of ring nebulae. This limits our understanding of the time-scales of formation and likely mass-loss rates. Following recent observational results noted above, simulations of ring nebulae involving both RSG and LBV ejections prior to a WR fast wind phase would also be of great use. 
Acknowledgments. The work in this presentation was funded in part by a grant from NASA administered by the AAS and NASA ADP grants NAG5-2999 and NAG5-6854.

\section{References}

Arnal, E.M., Cappa, C.E. 1996, MNRAS 279, 788

Arthur, S.J., Henney, W.J., Dyson, J.E. 1996, A\&A 313, 897

Cappa, C.E., Dubner, G.M., Rogers, C., St.-Louis, N. 1996, AJ 112, 1104

Cappa de Nicolau, C.E., Niemela, V.S., Dubner, G.M., Arnal, E.M. 1988, AJ 96, 1671

Chu, Y.-H. 1981, ApJ 249, 195

Chu, Y.-H. 1982, ApJ 254, 578

Chu, Y.-H. 1991, in: K.A. van der Hucht \& B. Hidayat (eds.), Wolf-Rayet Stars Interrelations with Other Massive Stars in Galaxies, Proc. IAU Symposium No. 143 (Dordrecht: Kluwer), p. 349

Chu, Y.-H., Treffers, R.R. 1981, ApJ 249, 586

Chu, Y.-H., Treffers, R.R., Kwitter, K.B. 1983, ApJS 53, 937

D'Ercole, A., 1992, MNRAS 255, 572

Dopita, M.A., Bell, J.F., Chu, Y.-H., Lozinskaya, T.A. 1994, ApJS 93, 455

Dubner, G.M., Niemela, V.S., Purton, C.R. 1990, AJ 99, 857

Dyson, J.E. 1989, in: G. Tenorio-Tagle, M. Moles \& J. Melnick (eds.), Structure and Dynamics of the Interstellar Medium, Proc. IAU Coll. No. 120 (Berlin: Springer), p. 137

Esteban, C., Vilchez, J.M. 1992, ApJ 390, 536

Esteban, C., Vilchez, J.M., Smith, L.J., Manchado, A. 1991, A\&A 244, 205

Esteban, C., Vilchez, J.M., Smith, L.J., Clegg, R.E.S. 1992, A\&A 259, 629

Esteban, C., Smith, L.J., Vilchez, J.M., Clegg, R.E.S. 1993, A\&A 272, 299

García-Segura, G.S., Mac Low M.-M., Langer, N. 1996a, A\&A 305, 229

García-Segura, G.S., Langer, N., Mac Low M.-M. 1996b, A\&A 316, 133

Goudis, C., Meaburn, J., Whitehead, M.J. 1988, A\&A 191, 341

Gruendl, R., Chu, Y.-H. 1998, BAAS 30, 830

Humphreys, R.M. 1991, in: K.A. van der Hucht \& B. Hidayat (eds.), Wolf-Rayet Stars Interrelations with Other Massive Stars in Galaxies, Proc. IAU Symposium No. 143 (Dordrecht: Kluwer), p. 485

Humphreys, R.M., Davidson, K. 1979, ApJ 232, 409

Kastner, J., Weintraub, D. 1996, ApJ 452, 833

Langer, N. 1991 A\&A 248, 531

Leitherer, C. 1997, in: A. Nota \& H. Lamers (eds.), Luminous Blue Variables: Massive Stars in Transition, ASP-CS 120, 58

Maeder, A. 1990, A\&AS 84, 139

Maeder, A., Meynet, A. 1994, A\&A 287, 803

Manchado, A., García-Segura, G.S., Chu, Y.-H. 1995, in: K.A. van der Hucht \& P.M. Williams (eds.) Wolf-Rayet Stars: Binaries, Colliding Winds, Evolution, Proc.

IAU Symp. No. 163 (Dordrecht: Kluwer), p. 74

Marston, A.P. 1991, ApJ 366, 81

Marston, A.P. 1995, AJ 109, 1839

Marston, A.P. 1996, AJ 112, 2828 
Marston, A.P. 1997, ApJ 475, 188

Marston, A.P., Meaburn, J. 1988, MNRAS 235, 391

Marston, A.P., Chu, Y.-H., García-Segura, G.S. 1994a, ApJS 93, 229

Marston, A.P., Yocum, D.R., García-Segura, G.S., Chu, Y.-H. 1994b, ApJS 95, 151

Marston, A.P., Welzmiller, J., Bransford, M., Black, J., Bergman, P. 1999a, ApJ in press

Marston, A.P., Nota, A., Pasquali, A., Clampin, M., Welzmiller, J., Schulte-Ladbeck, R. 1999b, in: B. Wolf, A.W. Fullerton \& O. Stahl (eds.), Variable and Non-spherical Stellar Winds in Luminous Hot Stars, Proc. IAU Coll. No. 169, Lecture Notes in Physics in press

Mathis, J.S., Cassinelli, J.P., van der Hucht, K.A., Prusti, T., Wesselius, P.R., Williams, P.M. 1992, ApJ 384, 197

Miller, G., Chu, Y.-H. 1993, ApJS 85, 137

Nichols, J.S., Fesen, R.A. 1994, A\&A 291, 283

Oudmaijer, R.D., Groenewegen, M.A.T., Matthews, H.E., Blommaert, J.A.D.L., Sahu, K.C. 1996, MNRAS 280, 1062

Schneps, M.H., Haschick, A.D., Wright, E.L., Barret, A.H. 1981, ApJ 243, 184

Smith, L.J. 1995, in: K.A. van der Hucht \& P.M. Williams (eds.) Wolf-Rayet Stars: Binaries, Colliding Winds, Evolution, Proc. IAU Symp. No. 163 (Dordrecht: Kluwer), p. 24

St-Louis, N., Doyon, R., Chagnon, F., Nadeau, D. 1998, AJ 115, 2475

Stothers, R.B., Chin, C.-w. 1996, ApJ 468, 842

Van Buren, D. 1986, ApJ 306, 538

\section{Discussion}

Maeder: In the case of anisotropic nebulae, like NGC 6888, would you consider that the anisotropics are related to the ejection processes or to some effect in the surrounding interstellar medium?

Marston: The interstellar medium has been swept clear of NGC 6888, so the shaping of the nebula is associated with the ejection processes rather than surrounding ISM.

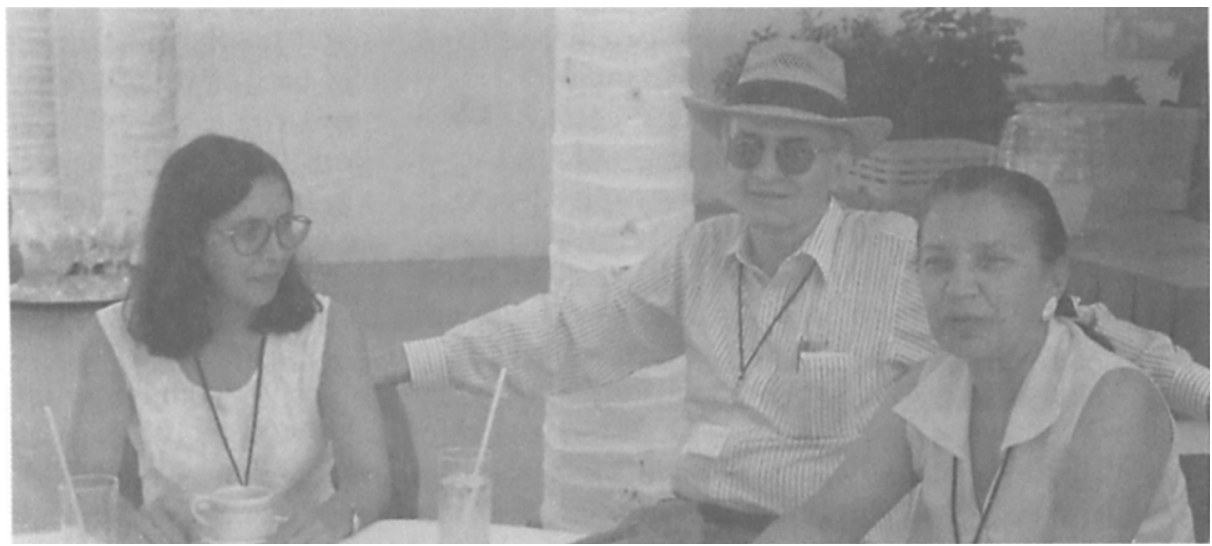

